\title{
Boundedness of convolution operators with smooth kernels on Orlicz spaces
}

\author{
by
}

Hugo Aimar, Eleonor Harboure, and Bibiana Iaffei (Santa Fe)

\begin{abstract}
We study boundedness in Orlicz norms of convolution operators with integrable kernels satisfying a generalized Lipschitz condition with respect to normal quasidistances of $\mathbb{R}^{n}$ and continuity moduli given by growth functions.
\end{abstract}

1. Introduction. In 1967 E. Stein and A. Zygmund, when dealing with the $L^{p}$ boundedness of translation invariant operators [10], proved that any convolution operator with integrable kernel $k$, satisfying a smoothness condition of the type

$$
\int_{\mathbb{R}^{n}}|k(x-y)-k(x)| d x \leq A|y|^{\delta}
$$

with $0<\delta<1$, maps $L^{p}$ into $L^{q}$ where $1 / q=1 / p-\delta / n$ and $1<p, q<\infty$. In terms of Taibleson-Lipschitz spaces those conditions on $k$ can be rephrased by saying that $k$ belongs to $\Lambda(\delta ; 1, \infty)$ [11]. Actually the main point in the $\mathrm{S}-\mathrm{Z}$ theorem is based on the harmonic extension characterization of $\Lambda(\delta ; 1, \infty)$ also given in [11]: $k \in \Lambda(\delta ; 1, \infty)$ if and only if the harmonic extension $k(x, t)=\left(k * P_{t}\right)(x)$ satisfies both

$$
\begin{gathered}
\int_{\mathbb{R}^{n}}|k(x, t)| d x \leq A, \\
\int_{\mathbb{R}^{n}}\left|\frac{\partial}{\partial t} k(x, t)\right| d x \leq A \frac{t^{\delta}}{t} .
\end{gathered}
$$

Several extensions and generalizations of the result of Stein and Zygmund are possible. First of all, the regularity condition (1.1) can be weakened to include continuity moduli more general than powers [4]. Second, the geometric radial control given by the absolute value of $y$ fails when dealing with

2000 Mathematics Subject Classification: Primary 47B38; Secondary 46E30.

Key words and phrases: translation invariant operators, Orlicz spaces.

The authors were supported by the Consejo Nacional de Investigaciones Científicas y Técnicas de la República Argentina and by the Universidad Nacional del Litoral, CAI+D Program. 
non-isotropic fractional integral operators on $\mathbb{R}^{n}$, but other distances can play the role of the absolute value.

The aim of this note is to approach at once both generalizations by giving an extension of the result of Stein and Zygmund to a more general setting. In this way, in Section 4 we obtain properties of functions in new spaces $\Lambda_{\varrho}(\eta ; 1, \infty)$, in terms of their convolutions with general families of approximate identities as considered in [12] and [1]. Although we do not obtain a complete characterization as in the classical case (Theorem 4, p. 421, of [11]) our result, Theorem 4.1, will be enough to prove the extension of the S-Z theorem to our general setting. This is done in Section 6 .

2. Generalization of a theorem of F. Zó. In [12], F. Zó shows that a non-negative integrable kernel $l$ defined on $\mathbb{R}^{n}$ satisfying the inequality

$$
|\nabla l(x)| \leq C|x|^{-n-1}
$$

for some finite constant $C$ and every $x \in \mathbb{R}^{n}-\{0\}$ defines by convolution with $l_{\varepsilon}(x)=\left(1 / \varepsilon^{n}\right) l(x / \varepsilon)$ an approximate identity with associated maximal operator of weak type $(1,1)$, even when it is not necessarily pointwise bounded by the Hardy-Littlewood maximal function. When dealing with a generalization of Zó's theorem to spaces of homogeneous type, in [1] it is proved that all radial kernels $l$ satisfying the above condition can be written as

$$
l(x)=\frac{\Phi\left(|x|^{n}\right)}{|x|^{n}}
$$

with $\Phi: \mathbb{R}^{+} \rightarrow \mathbb{R}^{+} \cup\{0\}$ satisfying

$$
\begin{gathered}
\Phi \in \mathcal{C}^{1}\left(\mathbb{R}^{+}\right) \cap L^{\infty}\left(\mathbb{R}^{+}\right), \\
\left|\Phi^{\prime}(s)\right| \leq C / s, \\
\int_{0}^{\infty} \frac{\Phi(s)}{s} d s<\infty .
\end{gathered}
$$

Since $l(x)$ depends only on $\varrho(x)=|x|^{n}$, the normalized quasi-distance induced on $\mathbb{R}^{n}$ by the usual distance $|x|$, our kernel can be rewritten in the form $l(x)=\Phi(\varrho(x)) / \varrho(x)$ and we can take $l_{t}(x)=\Phi(\varrho(x) / t) / \varrho(x)$. Parabolic metrics [2] or Vitali families on $\mathbb{R}^{n}$ [8] induce natural normal quasi-distances on $\mathbb{R}^{n}$. First of all let us remark that the general normalization of spaces of homogeneous type was obtained by Macías and Segovia [6], but we need a more refined normality condition. On the other hand since we are concerned with convolution operators our quasi-distances will be translation invariant. A translation invariant quasi-distance on $\mathbb{R}^{n}$ is a function $\varrho: \mathbb{R}^{n} \rightarrow \mathbb{R}^{+} \cup\{0\}$ such that

$$
\varrho(x)=0 \text { if and only if } x=0,
$$




$$
\varrho(x)=\varrho(-x) \text { for every } x \in \mathbb{R}^{n},
$$

there exists a constant $C$ such that

$$
\varrho(x+y) \leq C(\varrho(x)+\varrho(y)) \quad \text { for all } x \text { and } y \text { in } \mathbb{R}^{n} .
$$

We denote by $B_{\varrho}(x, r)$ the ball $\left\{y \in \mathbb{R}^{n}: \varrho(x-y)<r\right\}$; notice that $B_{\varrho}(x, r)=x+B_{\varrho}(0, r)$.

The quasi-distance $\varrho$ defines a structure of space of homogeneous type on $\mathbb{R}^{n}$ with the usual Lebesgue measure when the doubling condition

$$
\left|B_{\varrho}(0,2 r)\right| \leq A\left|B_{\varrho}(0, r)\right|
$$

holds true for some constant $A$ and every $r>0$. This is the case for the quasi-distances induced by non-isotropic dilations of $\mathbb{R}^{n}$ and, more generally, for regular Vitali families of neighborhoods of 0 in the sense of Riviere [8].

Let us now prove an auxiliary result that will be useful in our subsequent development.

LEMma 2.1. If $\delta$ is a translation invariant quasi-distance on $\mathbb{R}^{n}$ such that $\delta$ defines a structure of space of homogeneous type and the function $\mu(r)=\left|B_{\delta}(0, r)\right|$ is continuous on $\mathbb{R}^{+}$and strictly increasing, then

$$
\varrho(x)=\inf \left\{\left|B_{\delta}(0, r)\right|: x \in B_{\varrho}(0, r)\right\}
$$

defines a translation invariant quasi-distance such that every $\varrho$-ball is also $a \delta$-ball and $\left|B_{\varrho}(x, r)\right|=r$ for all $r$ and $x$.

Proof. First notice that since $\mathbb{R}^{n}=\bigcup_{r>0} B_{\delta}(0, r), \mu(r)$ tends to infinity as $r$ tends to infinity. Moreover, since the underlying measure is the usual Lebesgue measure, no atoms are allowed, hence $\mu\left(0^{+}\right)=0$. Let us now show that $\varrho$ is a quasi-distance on $\mathbb{R}^{n}$. Of course $\varrho(0)=0$ since $\mu\left(0^{+}\right)=0$; conversely, if $\varrho(x)=0$ then $x \in B_{\delta}(0, r)$ for every $r>0$ since $\mu(r)$ is assumed to be strictly increasing, thus $\delta(x)<r$ for every $r>0$, so that from (2.4) for $\delta$ we have $x=0$. Property (2.5) for $\varrho$ follows from the symmetry with respect the origin of $\delta$-balls. In order to check the triangle inequality, pick two points $x$ and $y$ in $\mathbb{R}^{n}$ and a positive number $\varepsilon$. Then there exist $r_{1}, r_{2}>0$ such that $x \in B_{\delta}\left(0, r_{1}\right), y \in B_{\delta}\left(0, r_{2}\right),\left|B_{\delta}\left(0, r_{1}\right)\right|<\varrho(x)+\varepsilon$ and $\left|B_{\delta}\left(0, r_{2}\right)\right|<\varrho(y)+\varepsilon$. Denote by $C_{\delta}$ the constant in (2.6) for $\delta$. Then $\delta(x, y) \leq C_{\delta}(\delta(x)+\delta(y))<$ $C_{\delta}\left(r_{1}+r_{2}\right)$. In other words $x+y \in B_{\delta}\left(0, C_{\delta}\left(r_{1}+r_{2}\right)\right)$. Then

$$
\begin{aligned}
\varrho(x+y) & \leq\left|B_{\delta}\left(0, C_{\delta}\left(r_{1}+r_{2}\right)\right)\right| \leq\left|B_{\delta}\left(0,2 C_{\delta} \max \left\{r_{1}+r_{2}\right\}\right)\right| \\
& \leq A_{\delta}^{1+\log 2 C_{\delta}}\left|B_{\delta}\left(0, \max \left\{r_{1}+r_{2}\right\}\right)\right| \leq A\{\varrho(x)+\varrho(y)\}+A \varepsilon,
\end{aligned}
$$

where $A_{\delta}$ is the constant in (2.7) for $\delta$ and $A=A_{\delta}^{1+\log 2 C_{\delta}}$. Since $\varepsilon$ is arbitrary 
we get (2.6) for $\varrho$ with $C=A$. Now, for $\delta>0$ we have

$$
\begin{aligned}
B_{\varrho}(0, s) & =\{x: \varrho(x)<s\} \\
& =\bigcup_{\left|B_{\delta}(0, r)\right|<s} B_{\delta}(0, r)=\bigcup_{\mu(r)<s} B_{\delta}(0, r)=B_{\delta}\left(0, \mu^{-1}(s)\right),
\end{aligned}
$$

hence $\left|B_{\varrho}(0, s)\right|=\left|B_{\delta}\left(0, \mu^{-1}(s)\right)\right|=s$.

Given a function $f$ defined on $\mathbb{R}^{n}$, we say that $f$ is $\varrho$-radial if $f(x)=$ $g(\varrho(x))$ for some function $g$ defined on the non-negative real numbers. The result of Lemma 2.1 allows us to compute integrals of radial functions on $\mathbb{R}^{n}$ as one-dimensional integrals. In fact,

$$
\int_{\mathbb{R}^{n}} f(x) d x=\int_{\mathbb{R}^{+}} g(t) d t
$$

provided that one of them exists.

Now applying the above mentioned results of [1] and [12] we have the next theorem about generalized radial approximate convolution identities.

TheOREM 2.2. Let $\varrho$ be a translation invariant quasi-distance on $\mathbb{R}^{n}$ such that $\left|B_{\varrho}(x, r)\right|=r$ for every $x$ in $\mathbb{R}^{n}$ and every positive $r$. Let $\Phi$ be a non-negative function defined on $\mathbb{R}^{+}$satisfying (2.1)-(2.3) normalized to have $\int_{0}^{\infty}(\Phi(t) / t) d t=1$. Then the family $\Phi(\varrho(x) / t) / \varrho(x)=l_{t}(x), t>0$, defines an approximate convolution identity in the following sense:

(i) The maximal operator $L f(x)=\sup _{t>0}\left|\left(l_{t} * f\right)(x)\right|$ satisfies a weak type $(1,1)$ inequality, that is, there exists a constant $C$ such that for every $\lambda>0$,

$$
\{x: L f(x)>\lambda\} \mid \leq \frac{C}{\lambda}\|f\|_{1} .
$$

(ii) The maximal operator $L$ is of strong type $(p, p)$ for $1<p \leq \infty$, that is,

$$
\|L f\|_{p} \leq C\|f\|_{p}
$$

for some constant $C$ and every measurable function $f$.

(iii) For $f$ in $L_{\text {loc }}^{1}\left(\mathbb{R}^{n}\right)$ and almost every $x \in \mathbb{R}^{n}$ we have the pointwise convergence $\left(l_{t} * f\right)(x) \rightarrow f(x)$ as $t \rightarrow 0^{+}$. If $f \in L^{p}, 1<p<\infty$, we also have the $L^{p}$ convergence.

In order to apply the above mentioned results of [1], we only need to observe that

$$
\begin{aligned}
\int_{\mathbb{R}^{n}} l_{t}(x) d x & =\int_{\mathbb{R}^{n}} \frac{\Phi(\varrho(x) / t)}{\varrho(x)} d x=\int_{0}^{\infty} \frac{\Phi(s / t)}{s} d s \\
& =\int_{0}^{\infty} \frac{\Phi(s)}{s} d s=1 .
\end{aligned}
$$


Let us point out that if $\Psi(s)$ is a standard basic shape for an approximate identity: $\Psi \in \mathcal{C}^{1}\left(\mathbb{R}^{+}\right), \operatorname{supp} \Psi \subset[0,1], \Psi$ non-increasing, $\Psi$ constant in a neighborhood of 0 and $\int \Psi(s) d s=1$, then taking $\Phi(s)=s \Psi(s)$ we get the result on approximate identities as a particular instance of this theorem of Zó.

Let us finally record a fact that will be used in Section 5 . Given a function $\Psi$ as described above and $l_{t}(x)=(1 / t) \Psi(\varrho(x) / t)$, it is easy to show that

$$
\left(l_{t} * l_{t}\right)(x) \leq \frac{3 C_{p}^{2}\|\Psi\|_{\infty}}{t} \chi_{B_{\varrho}\left(0,2 C_{\varrho} t\right)}
$$

Then the maximal operator $\sup _{t>0}\left|\left(l_{t} * l_{t} * k\right)(x)\right|$ is pointwise bounded by $M_{\varrho} k(x)$, the Hardy-Littlewood maximal function with respect to the $\varrho$-balls in $\mathbb{R}^{n}$. Since also $\int_{\mathbb{R}^{n}}\left(l_{t} * l_{t}\right)(x) d x=1$, we see that $\left(l_{t} * l_{t} * k\right)(x)$ is a good pointwise and $L^{p}$ approximation of $k(x)$.

3. Growth functions as continuity moduli. For completeness, we include in this section some usual notation and terminology (see [5]). We shall say that a non-negative, non-decreasing function $\eta$ defined on $\mathbb{R}^{+}$is a growth function if $\eta\left(0^{+}\right)=0$ and there exists a constant $A$ such that $\eta(2 t) \leq A \eta(t)\left(\Delta_{2}\right.$-condition $)$ for every $t>0$. We say that $\eta$ is of lower type $a \geq 0$ if

$$
\eta(s t) \leq C_{1} s^{a} \eta(t)
$$

for some constant $C_{1}$, every $0<s \leq 1$ and every $t>0$. Similarly $\eta$ is of upper type $b<\infty$ if

$$
\eta(s t) \leq C_{2} s^{b} \eta(t)
$$

for some constant $C_{2}$, every $s \geq 1$ and every $t>0$. Notice that the $\Delta_{2^{-}}$ condition is nothing but the condition of finite upper type. On the other hand, if $\eta$ is a growth function of positive lower type then $\eta(s) / s$ is integrable near zero, and moreover $\int_{0}^{t}(\eta(s) / s) d s \leq C \eta(t)$.

4. Generalized Taibleson's Lipschitz spaces $\Lambda_{\varrho}(\eta ; 1, \infty)$. Let $\varrho$ be a translation invariant quasi-distance on $\mathbb{R}^{n}$ such that $\left|B_{\varrho}(x, r)\right|=r$, and let $\eta$ be a growth function. We shall say that a function $k$ belongs to $\Lambda_{\varrho}(\eta ; 1, \infty)$ if

$$
\begin{aligned}
k & \in L^{1}\left(\mathbb{R}^{n}\right), \\
\int_{\mathbb{R}^{n}}|k(x-y)-k(x)| d x & \leq C \eta(\varrho(y)) \quad \text { for every } y \in \mathbb{R}^{n} .
\end{aligned}
$$

Given $k \in \Lambda_{\varrho}(\eta ; 1, \infty)$ and taking $l_{t}(x)=\Phi(\varrho(x) / t) / \varrho(x)$ as in Section 2, we consider the extension of $k$ to the upper half space $\mathbb{R}_{+}^{n+1}$ given by

$$
k(x, t)=\left(k * l_{t}\right)(x) .
$$


The main result of this section is the following necessary condition for a function to belong to $\Lambda_{\varrho}(\eta ; 1, \infty)$ in terms of $k(x, t)$.

THEOREM 4.1. Let $\eta$ be a growth function of positive lower type. Let $\Phi$ : $\mathbb{R}^{+} \rightarrow \mathbb{R}^{+} \backslash\{0\}$ with support in $[0,1]$ satisfy $(2.1)-(2.3)$ with $\int_{0}^{\infty}(\Phi(s) / s) d s=1$. If $k \in \Lambda_{\varrho}(\eta ; 1, \infty)$ then

$$
\begin{gathered}
\int_{\mathbb{R}^{n}}|k(x, t)| d x \leq A \\
\int_{\mathbb{R}^{n}}\left|\frac{\partial}{\partial t} k(x, t)\right| d x \leq C \frac{\eta(t)}{t},
\end{gathered}
$$

for some positive constants $A$ and $C$ and every $t>0$.

Proof. Of course (4.3) is nothing but a direct application of Young's inequality and the fact that $\int l_{t}(x) d x=1$. In order to prove (4.4) let us compute the derivative of $l_{t}(y)$ with respect to the parameter $t$ for fixed $y$ :

$$
\frac{\partial}{\partial t} l_{t}(y)=\frac{1}{\varrho(y)} \frac{\partial}{\partial t} \Phi\left(\frac{\varrho(y)}{t}\right)=-\frac{1}{t^{2}} \Phi^{\prime}\left(\frac{\varrho(y)}{t}\right) .
$$

On the other hand since $\int l_{t}(y) d y=1$, we have $\int \frac{\partial}{\partial t} l_{t}(y) d y=0$, so that applying (4.2) we obtain

$$
\begin{aligned}
\int_{\mathbb{R}^{n}}\left|\frac{\partial}{\partial t} k(x, t)\right| d x & =\int_{\mathbb{R}^{n}}\left|\int_{\mathbb{R}^{n}} \frac{\partial}{\partial t} l_{t}(y) k(x-y) d y\right| d x \\
& =\int_{\mathbb{R}^{n}}\left|\int_{\mathbb{R}^{n}} \frac{\partial}{\partial t} l_{t}(y)(k(x-y)-k(x)) d y\right| d x \\
& \leq \int_{\mathbb{R}^{n}} \int_{\mathbb{R}^{n}}\left|\frac{\partial}{\partial t} l_{t}(y)\right||k(x-y)-k(x)| d y d x \\
& \leq \int_{\mathbb{R}^{n}}\left|\frac{\partial}{\partial t} l_{t}(y)\right| \eta(\varrho(y)) d y .
\end{aligned}
$$

Now, from (2.2) we have

$$
\begin{aligned}
\int_{\mathbb{R}^{n}}\left|\frac{\partial}{\partial t} k(x, t)\right| d x & \leq \int_{B_{\varrho}(0, t)} \frac{1}{t^{2}} C \frac{t}{\varrho(y)} \eta(\varrho(y)) d y \\
& \leq \frac{C}{t} \int_{\mathbb{R}^{n}} \chi_{[0, t)}(\varrho(y)) \frac{\eta(\varrho(y))}{\varrho(y)} d y=\frac{C}{t} \int_{0}^{t} \frac{\eta(s)}{s} d s
\end{aligned}
$$

which in turn is bounded by a constant times $\eta(t) / t$, since $\eta$ is of positive lower type.

Actually, when $\varrho(x)=|x|^{n}$ is the normalization of the usual distance in $\mathbb{R}^{n}, l_{t}=P_{t}$ is the Poisson kernel and the function $\eta$ is also of upper type 
less than one, then the harmonicity of $k(x, t)$ allows one to show that for an integrable function $k,(4.4)$ is equivalent to $k \in \Lambda_{\varrho}(\eta ; 1, \infty)$ (see [4]).

5. The $L^{1}+L^{\infty}$ decomposition of a function $k \in \Lambda_{\varrho}(\eta ; 1, \infty)$. Let $k \in \Lambda_{\varrho}(\eta ; 1, \infty)$ with $\varrho$ and $\eta$ as in Section 4 and $\eta$ of upper type less than one. Take now $\Psi: \mathbb{R}^{+} \rightarrow \mathbb{R}^{+} \cup\{0\}$ of class $\mathcal{C}^{1}\left(\mathbb{R}^{+}\right)$, non-increasing, constant in a neighborhood of the origin and with $\int_{0}^{\infty} \Psi(s) d s=1$. From the remarks at the end of Section 2, we see that if we set $l_{t}(x)=(1 / t) \Psi(\varrho(x) / t)$, then both $l_{t} * k$ and $l_{t} * l_{t} * k$ are good approximate identities, both pointwise and in $L^{p}$ means for $1<p<\infty$. Write

$$
\bar{k}(x, t)=\left(l_{t} * l_{t} * k\right)(x)=\left(l_{t} * k(\cdot, t)\right)(x) .
$$

Given a positive number $\alpha$ we are going to pick a $t>0$ such that

$$
k(x)=(k(x)-\bar{k}(x, t))+\bar{k}(x, t)=k_{1}(x)+k_{\infty}(x),
$$

with $\left|k_{\infty}(x)\right|=|\bar{k}(x, t)| \leq \alpha$, and then we will estimate the $L^{1}$-norm of $k_{1}(x)$. Since $\bar{k}(x, t)=\left(l_{t} * k(\cdot, t)\right)(x)$, we have

$$
\sup _{x \in \mathbb{R}^{n}}|\bar{k}(x, t)| \leq\left(\sup _{y \in \mathbb{R}^{n}} l_{t}(y)\right) \int_{\mathbb{R}^{n}}|k(y, t)| d y \leq l_{t}(0)\|k\|_{1} \leq \frac{C}{t}\|k\|_{1} .
$$

Then

$$
\bar{k}(x, t)=-\int_{t}^{\infty} \frac{\partial}{\partial s} \bar{k}(x, s) d s=-\int_{t}^{\infty} 2\left[l_{s} * \frac{\partial}{\partial s} k(\cdot, s)\right](x) d s,
$$

from which we obtain

$$
|\bar{k}(x, t)| \leq 2 \int_{t}^{\infty} \sup _{x \in \mathbb{R}^{n}}\left|\left(l_{s} * \frac{\partial}{\partial s} k(\cdot, s)\right)(x)\right| d s \leq 2 \int_{t}^{\infty} \frac{C}{s}\left\|\frac{\partial}{\partial s} k(\cdot, s)\right\|_{1} d s .
$$

Applying (4.4) we deduce that

$$
\sup _{x \in \mathbb{R}^{n}}|\bar{k}(x, t)| \leq 2 C \int_{t}^{\infty} \frac{\eta(s)}{s^{2}} d s .
$$

Now, since $\eta$ is of upper type less than one the right hand side of the above inequality defines a decreasing one-to-one function $\widetilde{\eta}(t)$ onto $\mathbb{R}^{+}$(for details see [4]). Hence by choosing $t=\widetilde{\eta}^{-1}(\alpha)$ we have

$$
\left\|k_{\infty}(x)\right\|_{\infty} \leq \alpha .
$$

Let us now estimate $\left\|k_{1}(x)\right\|_{1}$ for this particular choice of $t$. By the remark at the end of Section 2 we have

$$
\bar{k}(x, t)-k(x)=\int_{0}^{t} \frac{\partial}{\partial s} \bar{k}(x, s) d s
$$


and consequently

$$
\begin{aligned}
\int_{\mathbb{R}^{n}}|\bar{k}(x, t)-k(x)| d x & \leq \int_{0}^{t} \int_{x \in \mathbb{R}^{n}}\left|\frac{\partial}{\partial s} \bar{k}(x, s)\right| d x d s=\int_{0}^{t} 2\left\|l_{s} * \frac{\partial}{\partial s} k(\cdot, s)\right\|_{1} d s \\
& \leq 2 \int_{0}^{t}\left\|l_{s}\right\|_{1}\left\|\frac{\partial}{\partial s} k(\cdot, s)\right\|_{1} d s .
\end{aligned}
$$

Since $\int l_{s}(x) d x=1$, from (4.5) we see, for $t=\widetilde{\eta}^{-1}(\alpha)$, that

$$
\int_{\mathbb{R}^{n}}\left|k_{1}(x)\right| d x \leq 2 \int_{0}^{\tilde{\eta}^{-1}(\alpha)} \frac{\eta(s)}{s} d s .
$$

So that we have the following $L^{1}+L^{\infty}$ decomposition of $k$.

LEMMA 5.1. Let $\varrho$ be a translation invariant quasi-distance on $\mathbb{R}^{n}$ with $\left|B_{\varrho}(0, r)\right|=r$. Let $\eta$ be a non-negative function of positive lower type and upper type less than one. Let $k \in \Lambda_{\varrho}(\eta ; 1, \infty)$ and $\alpha>0$. Then $k$ can be written as

$$
k(x)=k_{1}(x)+k_{\infty}(x)
$$

with

$$
\left\|k_{\infty}\right\| \leq \alpha, \quad\left\|k_{1}\right\|_{1} \leq 2 \int_{0}^{\tilde{\eta}^{-1}(\alpha)} \frac{\eta(s)}{s} d s, \quad \widetilde{\eta}(t)=2 C \int_{t}^{\infty} \frac{\eta(s)}{s^{2}} d s .
$$

6. Generalized Stein-Zygmund theorem. In this section we sketch the proof of the main result of this note concerning the boundedness in Orlicz spaces of a convolution operator with kernel in $\Lambda_{\varrho}(\eta ; 1, \infty)$.

In what follows, by a Young function $\Theta$ we shall mean a non-negative, convex non-decreasing function defined on $[0, \infty]$ with $\Theta(0)=0, \Theta(\infty)=\infty$ but neither identically zero nor identically infinity. We notice that $\Theta$ may have a jump at some $x_{1}>0$, but in this case $\lim _{x \rightarrow x_{1}^{-}} \Theta(x)=\infty$ and $\Theta(x)=\infty$ for $x \geq x_{1}$. Under these assumptions the inverse function $\Theta^{-1}$ is well defined and it is also increasing and continuous.

For a Young function $\Theta$ we define the Orlicz space $L_{\Theta}=L_{\Theta}(X)$ as the linear space of those measurable functions defined on the measure space $(X, \mu)$ for which there is a finite number $K>0$ such that

$$
\int_{X} \Theta(|f(x)| / K) d \mu<\infty .
$$

The infimum of such constants $K$ is a norm which will be denoted by $\|f\|_{\Theta}$.

TheOREM 6.1. Let $\varrho$ be a translation invariant quasi-distance in $\mathbb{R}^{n}$ such that $\left|B_{\varrho}(0, r)\right|=r$. Let $\eta$ be a growth function of positive lower type 
and upper type less than one. Let $T$ be the convolution operator with kernel $k \in \Lambda_{\varrho}(\eta ; 1, \infty)$. Then, for every Young function $\Theta$ satisfying

$$
r \Theta(s) \leq s \Theta^{\prime}(s)
$$

for some $r>1$ and every $s>0$, and

$$
\int_{0}^{1} \frac{\eta\left(t^{-1}\right) \Theta^{-1}(t)}{t} d t<\infty
$$

$T$ is a bounded linear operator from $L_{\Theta}$ in $L_{\Omega}$, where

$$
\Omega^{-1}(s)=\int_{0}^{s} \frac{\eta\left(t^{-1}\right) \Theta^{-1}(t)}{t} d t .
$$

Moreover, there exists a constant $C$ independent of the $L^{1}$ norm of $k$ such that, for every measurable function $f$,

$$
\|T f\|_{\Omega} \leq C\|f\|_{\Theta}
$$

The result of Section 5 provides us the basic tool for estimating the size of the distribution function of the kernel $k$, leading us to show that $k$ belongs to an appropriate weak Orlicz space. This fact will finally allow us to apply a result of O'Neil [7] completing the proof of Theorem 6.1.

We consider a weak version of Orlicz spaces, namely, for a Young function $\Theta$, a measurable function $f$ is said to belong to the weak Orlicz class defined by $\Theta$ if there exists a constant $C$ such that

$$
\Theta(\alpha)|\{x:|f(x)|>\alpha\}| \leq C \quad \text { for every positive } \alpha .
$$

Lemma 6.2. Let $\varrho, \eta$ and $k$ be as in Theorem 6.1. Then $k$ belongs to the weak Orlicz space given by a Young function $\widetilde{\zeta}$ equivalent to

$$
\zeta(\alpha)=\frac{\alpha}{\int_{0}^{\widetilde{\eta}^{-1}(\alpha)} \frac{\eta(s)}{s} d s} .
$$

In other words

$$
\widetilde{\zeta}(\alpha)|\{x:|k(x)|>\alpha\}| \leq C
$$

for some constant $C$ independent of the $L^{1}$ norm of $k$ and every positive $\alpha$.

Proof. The function $\zeta$ defined by (6.2) has all the properties of a Young function except, perhaps, the convexity. Nevertheless

$$
\widetilde{\zeta}(\alpha)=\int_{0}^{\alpha} \frac{\zeta(s)}{s} d s
$$

is a Young function which is equivalent to $\zeta$ in the sense that $\widetilde{\zeta} / \zeta$ is bounded above and below. For a proof of this fact we refer to [4]. Take $\alpha>0$ and 
apply Lemma 5.1 to obtain

$$
\begin{aligned}
|\{x:|k(x)|>2 \alpha\}| & \leq\left|\left\{x:\left|k_{1}(x)\right|>\alpha\right\}\right|+\left|\left\{x:\left|k_{\infty}(x)\right|>\alpha\right\}\right| \\
& \leq\left|\left\{x:\left|k_{1}(x)\right|>\alpha\right\}\right| \leq C \frac{1}{\alpha} \int_{0}^{\widetilde{\eta}^{-1}(\alpha)} \frac{\eta(s)}{s} d s .
\end{aligned}
$$

We next state a slight modification of a theorem of [7] on fractional integration for Orlicz spaces. Let us first recall the definition of the distribution and rearrangements of functions. For a function $f$ on $\mathbb{R}^{n}$, the non-increasing rearrangement of $f$, denoted by $f^{*}$, is the generalized inverse of the distribution of $f$, in other words

$$
f^{*}(s)=\inf \left\{t: \mu_{f}(t) \leq s\right\} \quad \text { where } \mu_{f}(t)=|\{x:|f(x)|>t\}| .
$$

We denote by $f^{* *}(t)$ the average of $f^{*}$ over the interval $[0, t]$, that is,

$$
f^{* *}(t)= \begin{cases}t^{-1} \int_{0}^{t} f^{*}(s) d s & \text { if } t>0 \\ f^{*}(0) & \text { if } t=0 .\end{cases}
$$

Following O'Neil [7], we say that for a given function $\zeta$ equivalent to a Young function, the measurable function $f$ defined on $\mathbb{R}^{n}$ belongs to $M_{\zeta}$ if there exists a constant $C$ such that

$$
f^{* *}(t) \leq C \zeta^{-1}(1 / t) \quad \text { for every } t>0 .
$$

Let us remark that a "weak" inequality like

$$
\zeta(\alpha)|\{|k(x)|>\alpha\}| \leq C
$$

is generally not enough for $k$ to belong to $M_{\zeta}$. But, as shown in [3], both conditions are equivalent if and only if $\zeta$ is of lower type larger than one.

TheOREm 6.3. Let $\zeta$ be an increasing function such that $\zeta^{-1}(t) / t$ is non-increasing. Let $k$ be such that $k^{* *}(t) \leq C \zeta^{-1}(1 / t)$. Let $\Theta$ be a Young function satisfying

$$
r \Theta(t) \leq t \Theta^{\prime}(t)
$$

for some $r>1$ and every $t>0$, and

$$
\int_{0}^{1} \frac{\zeta^{-1}\left(t^{-1}\right) \Theta^{-1}(t)}{t^{2}} d t<\infty .
$$

Then the operator $T(f)=k * f$ is bounded from $L_{\Theta}$ into $L_{\Omega}$, where

$$
\Omega^{-1}(s)=\int_{0}^{s} \frac{\zeta^{-1}\left(t^{-1}\right) \Theta^{-1}(t)}{t^{2}} d t .
$$

Now we are in a position to complete the proof of the generalized SteinZygmund theorem stated at the beginning of this section. 
Proof of Theorem 6.1. It is not hard to show that $\zeta^{-1}(t)$ and $t \eta\left(t^{-1}\right)$ are equivalent functions (for details see [4]). From Lemma 6.2 we know that

$$
\zeta(\alpha)|\{x:|k(x)|>\alpha\}| \leq C .
$$

By the above remark, this inequality implies $k^{* *}(t) \leq C \zeta^{-1}(1 / t)$ whenever $\zeta$ is of lower type larger than one. But the latter is actually a consequence of $\zeta^{-1}(t) \sim t \eta(1 / t)$, and the fact that $\eta$ is of positive lower type. To finish the proof of the theorem, we notice that

$$
\int_{0}^{s} \frac{\zeta^{-1}\left(t^{-1}\right) \Theta^{-1}(t)}{t^{2}} d t \sim \int_{0}^{s} \frac{\eta\left(t^{-1}\right) \Theta^{-1}(t)}{t} d t,
$$

and the result follows by applying Theorem 6.3.

Typical classical examples of Stein-Zygmund operators are the fractional integral operators of order $\alpha$ or Riesz potentials, of which the Newtonian potentials are special cases:

$$
I_{\alpha} f(x)=\int \frac{|x-y|^{\alpha}}{|x-y|^{n}} f(y) d y, \quad 0<\alpha<n .
$$

If we consider the standard normalization of $\mathbb{R}^{n}, \varrho(x-y)=|x-y|^{n}$, the operator takes the form

$$
I_{\alpha} f(x)=\int \frac{(\varrho(x-y))^{\alpha / n}}{\varrho(x-y)} f(y) d y, \quad 0<\alpha<n,
$$

which makes sense for every quasi-distance of the type considered in this paper. Moreover if the power function $\eta(t)=t^{\alpha / n}$ is replaced by a function $\eta$ like those considered in Theorem 6.1 , we arrive at the following generalization of the Riesz potentials:

$$
I_{\eta}^{\varrho} f(x)=\int_{\mathbb{R}^{n}} \frac{\eta(\varrho(x-y))}{\varrho(x-y)} f(y) d y .
$$

As a straightforward application of our result we deduce that the truncations of the above operator are bounded from $L_{\Theta}$ into $L_{\Omega}$ where $\Theta$ and $\Omega$ are as in Theorem 6.1. Now, since the constant $C$ in the norm inequality

$$
\|T f\|_{\Omega} \leq C\|f\|_{\Theta}
$$

is independent of the $L^{1}$ norm of the kernel and the truncated kernels satisfy the regularity property in (4.2) uniformly, the $L_{\Theta} \rightarrow L_{\Omega}$ boundedness of $I_{\eta}^{o}$ follows.

\section{References}

[1] H. Aimar, Singular integrals and approximate identities on spaces of homogeneous type, Trans. Amer. Math. Soc. 292 (1985), 135-153. 
[2] M. de Guzmán, Real Variable Methods in Fourier Analysis, North-Holland Math. Stud. 46, North-Holland, 1981.

[3] B. Iaffei, Comparison of two weak versions of Orlicz spaces, Rev. Uni. Mat. Argentina 40 (1996), 191-202.

[4] - Espacios de Lipschitz generalizados y operadores invariantes por traslaciones, Tesis Doctoral, Univ. Nacional del Litoral, Santa Fe, 1997.

[5] S. Janson, Generalizations of Lipschitz spaces and an application to Hardy spaces and bounded mean oscillation, Duke Math. J. 47 (1980), 959-982.

[6] R. Macías and C. Segovia, Lipschitz functions on spaces of homogeneous type, Adv. Math. 33 (1979), 257-270.

[7] R. O'Neil, Fractional integration in Orlicz spaces, Trans. Amer. Math. Soc. 115 (1965), 300-328.

[8] N. Riviere, Singular integrals and multiplier operators, Ark. Mat. 9 (1973), 243-270.

[9] E. Stein, Singular Integrals and Differentiability Properties of Functions, Princeton Univ. Press, 1970.

[10] E. Stein and A. Zygmund, Boundedness of translation invariant operators on Hölder spaces and $L^{p}$-spaces, Ann. of Math. 85 (1967), 337-349.

[11] M. Taibleson, On the theory of Lipschitz spaces of distributions on Euclidean $n$ space. I. Principal properties, J. Math. Mech. 13 (1964), 407-479.

[12] F. Zó, A note on the approximation of the identity, Studia Math. 55 (1976), 111-122.

Departamento de Matemática

FIQ-IMAL

Universidad Nacional del Litoral

CONICET

Santa Fe, Argentina

E-mail: haimar@ceride.gov.ar

harbour@ceride.gov.ar
Departamento de Matemática

FHUC-IMAL

Universidad Nacional del Litoral

CONICET

Santa Fe, Argentina

E-mail: biaffei@math.unl.edu.ar

Received November 8, 1999

Revised version January 2, 2002 